\title{
Factors that Affect Development of Collar Rot on Tobacco Seedlings Grown in Greenhouses
}

\author{
W. A. Gutierrez and H. D. Shew, Department of Plant Pathology, Box 7616, North Carolina State University, \\ Raleigh 27695
}

\begin{abstract}
Gutierrez, W. A., and Shew, H. D. 2000. Factors that affect development of collar rot on tobacco seedlings grown in greenhouses. Plant Dis. 84:1076-1080.

Collar rot, caused by Sclerotinia sclerotiorum, is an important disease of tobacco transplants produced under greenhouse conditions. Factors that affect the development of the disease were studied, including age of seedlings, presence of an external source of nutrients (leaf extract), clipping (leaf removal) practices, exposure to low temperature, and leaf injury caused by heat and a chemical. Flue-cured tobacco seedlings, cultivar K-326, were grown in polystyrene cell trays floating on a nutrient solution. Trays were maintained in a phytotron growth chamber with a $28 / 18^{\circ} \mathrm{C}$ day/night temperature regime. Seedlings were inoculated with ascospores of S. sclerotiorum at the desired stage of growth by placing mature apothecia in the growth chamber and inducing ascospore release and deposition. Disease incidence was determined by examining seedlings for the presence of stem lesions over the next 15 to 21 days. Seedlings between 35 and 53 days old were more susceptible to collar rot than younger or older seedlings. Inoculum efficiency was highest and disease was most severe when an external source of nutrients was present on leaf surfaces. Clipping of leaf tips did not increase disease, but if the leaf pieces created by clipping were left on seedlings, collar rot development was enhanced compared with treatments where debris was removed. Heat and chemical injuries that resulted in necrotic tissue provided highly susceptible infection courts for ascospores of S. sclerotiorum, but exposure to low temperature, which caused no visible injury, did not enhance infection. Adoption of cultural practices that minimize accumulation of leaf debris and eliminate factors that cause necrotic injury on leaves should greatly reduce the severity of collar rot of tobacco seedlings.
\end{abstract}

Additional keywords: apothecium production, ascospore inoculation

Collar rot, caused by Sclerotinia sclerotiorum, is a potentially severe disease of tobacco (Nicotiana tabacum) transplants produced in greenhouses. Ascospores are the primary inoculum for this disease and are produced around tobacco greenhouses throughout the transplant production period, February through April, in North Carolina (15). Ascospores enter greenhouses through open side vents and often reach very high numbers in the morning hours (15). Symptoms of the disease, however, are observed only after a closed canopy has formed within a seedling tray (15).

Most flue-cured tobacco seedlings are produced in greenhouses using a float-tray system. The tobacco production season in North Carolina starts in early spring, when polystyrene trays are filled with a soilless medium and seeded with pelletized tobacco seed. On average, seedlings are grown in the greenhouse for about 60 to 70 days, then removed and transplanted into

Corresponding author: H. D. Shew

E-mail: david_shew@ncsu.edu

Accepted for publication 8 June 2000 .

Publication no. D-2000-0727-01R

(C) 2000 The American Phytopathological Society the field. Production of uniform transplants requires that seedlings be clipped (mowed) multiple times (30). Clipping removes leaf tissue about 3 to $4 \mathrm{~cm}$ above the bud and increases uniformity in plant height and stem diameter, but it also may result in the accumulation of large amounts of leaf debris left on seedlings (30).

Various factors affect development of Sclerotinia diseases under field conditions $(4,6,8,15,23,26)$. The ability of $S$. sclerotiorum to infect and colonize host tissues depends upon the type of inoculum present, the nutrient status of the fungus, properties of the host, and prevailing environmental conditions $(5,11,21,24,28,35,36)$. For example, ascospores required free water and an external source of nutrients for host penetration $(2,12,26)$, and Grogan and Abawi (12) concluded that leaf tissues must remain wet for a minimum period of about $16 \mathrm{~h}$ at $25^{\circ} \mathrm{C}$ in order for infection to take place.

The recent change from a field-based to a greenhouse-based transplant production system has changed collar rot from a minor problem (20) to one of the most important problems in the production of tobacco transplants. Lack of information about factors that affect the development of collar rot under greenhouse conditions has made control of this disease difficult. The objective of this study was to determine how various production practices affect the development of collar rot of tobacco seedlings grown in greenhouses using the floattray system. Factors studied included age of seedlings, presence of an external source of nutrients (leaf extract), clipping (leaf removal) practices, exposure to low temperature, and leaf injury caused by high heat and a chemical.

\section{MATERIALS AND METHODS}

Growth conditions. Flue-cured tobacco seedlings, cultivar K-326, were grown in polystyrene trays in a phytotron chamber $(2.0 \times 4.5 \times 2.1 \mathrm{~m})$ at $28 / 18^{\circ} \mathrm{C}$ day/night temperatures and a $12-\mathrm{h}$ photoperiod. Polystyrene trays $(11 \times 15$ cells, each cell $2.4 \times$ $2.4 \mathrm{~cm}$ ) were filled with a soilless potting mix and seeded with pelletized tobacco seeds. Seeded trays were placed into polyethylene pans (Nalgene; $54.1 \times 43.5 \times 14.3$ $\mathrm{cm})$ that were lined with two folds of 1.1mil black plastic and filled with water to simulate the greenhouse float-tray system used by tobacco growers. Water soluble fertilizer (20-20-20, Spectrum Group, Division of Industries Corporation, St. Louis, MO) was added at $150 \mathrm{mg} /$ liter of water 7 days after seeding, and an additional 75 $\mathrm{mg} / \mathrm{liter}$ was added 14 days later (30). Seedlings were grown under these conditions until they reached the desired age for inoculation. Seedlings were misted daily beginning 2 days before inoculation and for 15 days after inoculation to simulate the high relative humidity present in greenhouses in early morning hours. Seedlings were misted over a $30-\min$ period (15 $\mathrm{s}$ of mist every $5 \mathrm{~min}$ for a total of seven mist periods) before lights were turned on in the growth chamber in the morning. The misting period created a relative humidity between 95 and $99 \%$ and resulted in the presence of water on leaves.

Inoculation of plants. Ascospores of a tobacco isolate of $S$. sclerotiorum were used as inoculum in all tests. To induce apothecia, sclerotia (2 to $4 \mathrm{~mm}$ diameter) were placed into $118-\mathrm{ml}$ glass jars (Qorpak, Fisher Scientific Co., Norcross, GA) containing $50 \mathrm{~cm}^{3}$ of sterile sand and water $(4: 1 \mathrm{vol} / \mathrm{vol})$. Ten sclerotia were placed on the sand surface in each jar. Jars were covered with a $60 \times 15 \mathrm{~mm}$ plastic petri dish lid, and the lid was sealed with Parafilm. Jars were incubated at $4^{\circ} \mathrm{C}$ in the dark for 30 days, and moved to $12^{\circ} \mathrm{C}$ until stipes were visible (5 $\mathrm{mm}$ long). When 
stipes were visible, jars were moved to a growth chamber at $16+/-2^{\circ} \mathrm{C}$ with $12 \mathrm{~h}$ of light until apothecia were fully developed (14). Six jars, each containing mature apothecia, were used to inoculate seedlings in the growth chamber. Jars with mature apothecia were placed at six points in the growth chamber and opened to release ascospores on four consecutive days. Jars were elevated to approximately $30 \mathrm{~cm}$ above the seedling canopy and opened for 30 to $45 \mathrm{~s}$ to allow ascospore release. This procedure was repeated after $1 \mathrm{~h}$ (two releases from each jar on each of the 4 days).

Seeding age. Trays were seeded at 7to 8-day intervals to establish seedlings of six different ages. Seedlings were inoculated when they were $22,30,38,45,52$, and 60 days old. Trays were arranged in a randomized block design of six treatments (ages) with three replications (trays) per treatment. The test was conducted twice.

Inoculum concentration, leaf extract, and plant age. Ascospores were collected following the protocol described by Steadman and Cook (31), quantified, and added to sterile deionized water to establish spore suspensions of either 800 or 8,000 ascospores per ml. Leaves on seedlings $25,35,45$, and 60 days old were inoculated by adding a 50- $\mu$ d drop of the appropriate ascospore suspension to either a $50-\mu 1$ drop of water or leaf extract already present on the leaf surface. This resulted in either 400 or 4,000 ascospores per inoculation site. Treatments, seedling age, inoculum concentration, and leaf extract (+/-) were arranged in a factorial design with three replications per treatment. The experimental design was a split plot, with age of seedlings as main plots and the combination of inoculum concentration and leaf extract as subplots. Each replication consisted of a tray with seedlings of a given age, and the tray was divided into four sections, each section containing 35 cells. The test was conducted twice.

Clipping effect. Seedlings 35 days old were clipped before or after inoculation. Clipping consisted of leaf tip removal (1/3 to $1 / 2$ of each leaf) with scissors when the seedlings were about 10 to $12 \mathrm{~cm}$ in height. Treatments included: (i) a no-clipping control, (ii) clipping and removal of leaf debris before inoculation, (iii) clipping before inoculation and leaving leaf debris, (iv) clipping and removal of leaf debris after inoculation, and (v) clipping after inoculation and leaving leaf debris on seedling surfaces. To simulate the effect of the clipping devices (modified lawn mowers) used in greenhouses, clipped leaf pieces were ground using a mortar and pestle and then distributed over their respective seedling trays. The test was conducted twice.

Effect of leaf injury. Seven days before inoculation, 30-day-old tobacco seedlings were exposed to several types of injury that are commonly observed in tobacco greenhouses. Treatments included: (i) no injury, (ii) chemical injury accomplished by spraying leaves with $5 \mathrm{ml}$ of methanol (99\%) per tray, (iii) heat injury accomplished by blowing dry, hot air across seedlings with a commercial air dryer for 2 min, and (iv) exposure to low temperature $\left(4^{\circ} \mathrm{C}\right)$ for $14 \mathrm{~h}$ by placing trays into a cold chamber overnight. Seedlings were inoculated as previously described, and the test was conducted twice.

Disease evaluations. Seedlings were observed daily after inoculation for collar rot development. Final incidence of collar rot (percentage of infected plants per tray) was recorded 15 days after inoculation for all tests except the seedling age test, for which readings were recorded daily for 21 days after inoculation.

The concentration of $S$. sclerotiorum ascospores released from apothecia was assessed using a semi-selective medium (13). Fifteen 9-cm-diameter petri dishes containing the semi-selective medium were placed throughout the growth chamber, and the lids were removed for a 2-h period after ascospore release. After 3 days of incubation in the dark at room temperature (22 to $25^{\circ} \mathrm{C}$ ), the number of ascospores (colonies) of $S$. sclerotiorum deposited on the dishes was counted.

Except where noted, all tests were conducted in a randomized complete block design and were conducted in the same large growth chamber. Data were analyzed by the PROC GLM procedures of SAS (SAS Institute Inc., Cary, NC), and significant differences were determined by the Waller-Duncan $k$-ratio $t$ test. Data were combined across runs of all experiments, based on statistical analyses and homogeneity of variances, and data presented are means across runs.

\section{RESULTS}

Seedlings grown in the phytotron chamber were similar in size and appearance to those observed in commercial greenhouses over the 60-day duration of experiments. Seedlings emerged within 7 to 10 days, and a closed canopy was evident 28 to 35 days after seeding.

Trapping of ascospores indicated no significant differences in ascospore numbers between runs of experiments (average 13 to 15 ascospores per petri dish). Differences in numbers were observed between the two ascospore releases on a given day, with the second release about four times greater than the first release. Thus, two release periods per day were used in all experiments using apothecia.

Seedling age. Seedling age had a significant effect on incidence and development of collar rot. Seedlings 45 and 52 days old at the time of inoculation had the highest incidence of collar rot (44 to 50\%), followed by 38 - and 60 -day-old seedlings
(12 to 17\%) (Fig. 1A). The lowest incidence of collar rot was observed on 22and 30-day-old seedlings (Fig. 1A). Incubation period also differed with plant age. Symptoms of collar rot were observed 7 to 9 days after inoculation on 38- to 60-dayold seedlings, but required 12 days for 30 day-old seedlings and 20 days for 22-dayold seedlings (Fig. 1B).

Inoculum concentration, leaf extract, and plant age. Inoculum concentration, presence of leaf extract, and plant age significantly affected collar rot development $(P<0.001)$. In addition, highly significant interactions $(P<0.001)$ were observed between leaf extract and inoculum level and between leaf extract and plant age. Leaf extract, inoculum concentration, and their interaction accounted for about $91 \%$ of the total sums of squares in the ANOVA. Plant age and the leaf extract by plant age interaction accounted for most $(8 \%)$ of the remaining variation observed in the experiment.

The presence of leaf extract greatly increased inoculum efficiency (Fig. 2). For example, on 35-day-old seedlings, disease incidence was only $1 \%$ when 400 ascospores were added in water to an inoculation site, but $16 \%$ when 400 ascospores were added in leaf extract. When 4,000 ascospores were added per inoculation site, disease increased from $2 \%$ with ascospores added in water to $36 \%$ with ascospores added in leaf extract (Fig. 2). Similar trends were observed with other plant ages except the 60-day-old seedlings (Fig. 2).

Susceptibility of plant tissues to $S$. sclerotiorum increased with age of seedlings up to 45 days old, but then decreased sharply in all treatment combinations on 60-day-old seedlings except for the high inoculum level in water (Fig. 2). For example, when 4,000 ascospores were added in leaf extract, disease incidence was $30 \%$ on 25-day-old seedlings, $36 \%$ on 35-dayold seedlings, and $50 \%$ on 45-day-old seedlings, but only $25 \%$ on 60 -day-oldseedlings. The highest disease incidence observed (50\%) occurred when ascospores were placed in leaf extract on 45-day-old seedlings; the highest incidence observed when ascospores were placed in water (17\%) occurred on 60-day-old seedlings. The three-way interaction was not significant $(P<0.09)$.

A significant interaction of inoculum concentration by leaf extract was observed for incubation period; no other variables affected incubation period. With 400 spores per inoculation site, incubation period was similar in water and leaf extract. With 4,000 spores per inoculation site, incubation period was decreased by 2 days by placing spores in leaf extract.

Clipping effect. There was no effect of clipping, compared with not clipping, on the incidence of collar rot if the clipped debris was removed from plants (Fig. 3A). When clipped debris was left on seedlings, 
a significant increase in collar rot was observed (Fig. 3B).

Effect of leaf injury. The injury to leaves caused by heat and methanol was characterized by the development of necrotic spots at leaf edges and in interveinal areas. Necrotic lesions were observed 3 days after treatment. No symptoms were observed on seedlings exposed to the low temperature treatment. Incidence of collar rot was $65 \%$ on seedlings injured by heat and $62 \%$ on seedlings injured by methanol, compared with only $13 \%$ in control seedlings and $10 \%$ on seedlings exposed to low temperature (Fig. 4). The incubation period also was less in seedlings injured by heat (6 days) or methanol (5 days) than in the control (10 days) or low temperature treatments (12 days).

\section{DISCUSSION}

This study investigated the effects of host and environmental factors on the incidence and development of collar rot of tobacco. Because no fungicides are currently labeled for control of this disease in the greenhouse, emphasis in disease management has shifted to elimination of factors that enhance or encourage disease development. We identified host and envi- ronmental factors that enhance disease incidence, and elimination or management of these factors should complement previous recommendations designed to reduce inoculum levels in greenhouses (15).

The presence or absence of leaf extract at infection courts was the most important factor in determining incidence of collar rot. When known inoculum densities were added to leaf surfaces, the presence of leaf extract greatly increased collar rot incidence and severity. These results are in agreement with previous studies of the effects of an external source of nutrient on ascospore germination and infection of host tissues $(3,17,23,33,35)$. The greatest effect of leaf extract was on young seedlings. Little or no disease developed on seedlings less than 35 days old at the time of inoculation unless leaf extract was present, even when high inoculum levels were present. Therefore, growers would not need to apply chemical controls, if they become available, prior to this time.

The important role of leaf extract in disease development was supported by results from the clipping experiments. Tobacco seedlings are typically clipped three to five times during the production period to increase plant uniformity at transplanting

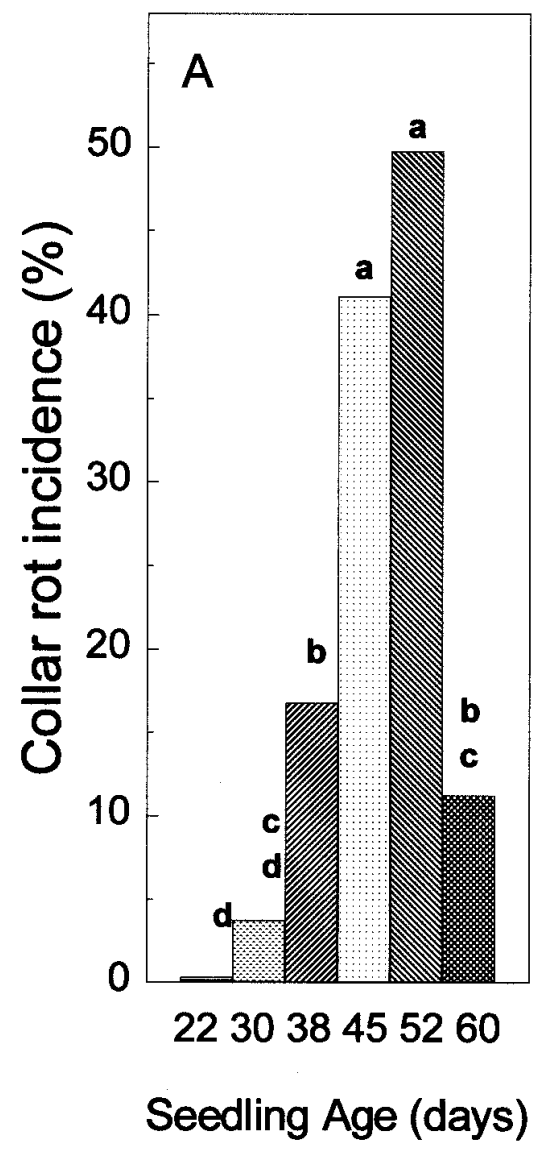

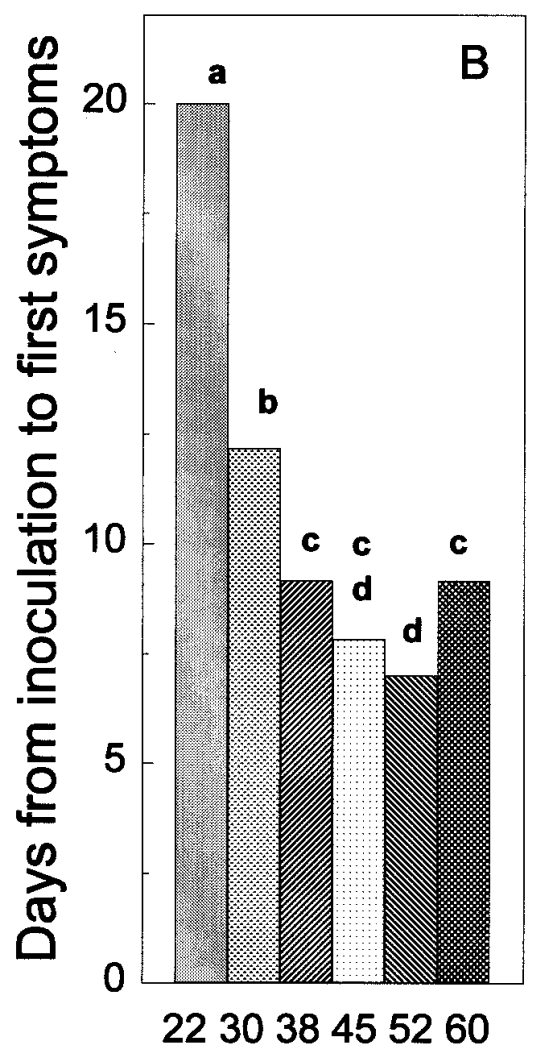

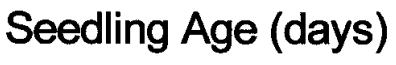

Fig. 1. (A) Influence of seedling age at time of inoculation on incidence of collar rot of tobacco. Seedlings were grown in polystyrene trays floated on water and inoculated with ascospores of the pathogen released from apothecia. (B) Number of days after inoculation required for symptom development on tobacco seedlings. Bars with different letters are significantly different $(P=0.05)$ according to the Waller-Duncan $k$-ratio test, $k=100$. 
development of Sclerotinia on tobacco under field conditions, with leaves becoming more susceptible with age. We also observed lowest levels of disease in young seedlings, but in contrast to the previous study, 60-day-old seedlings had less disease than 35- or 45-day-old seedlings in this study. There appeared to be a threshold
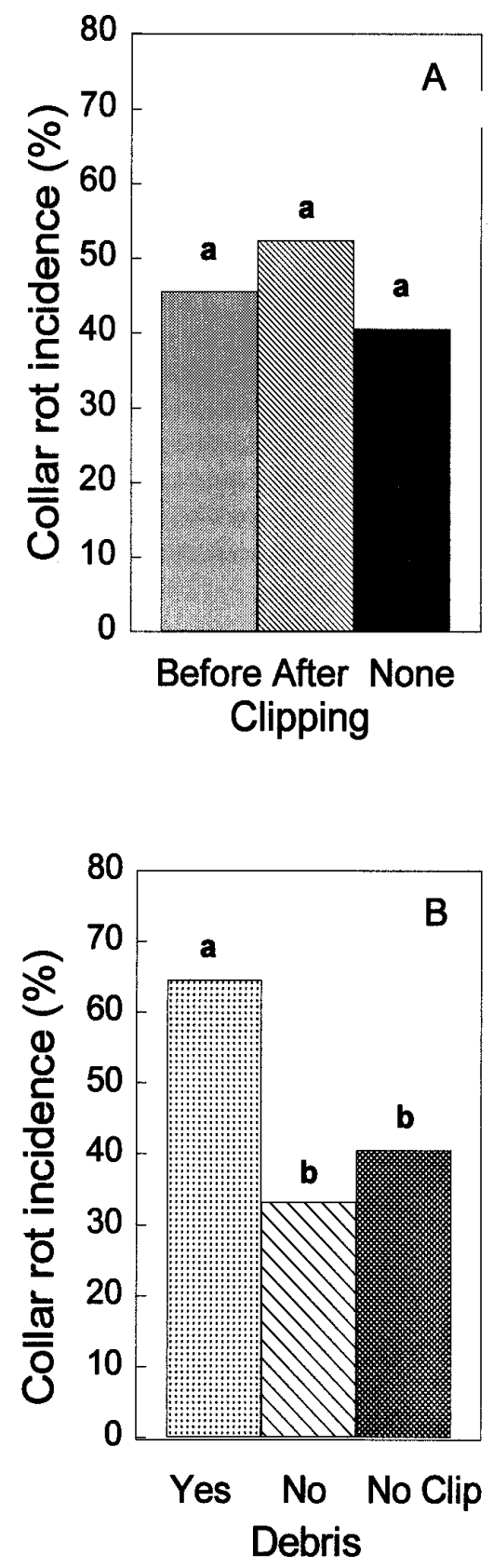

Fig. 3. Effect of clipping practices on the incidence of collar rot of tobacco seedlings grown in float trays. (A) Effect of clipping before or after inoculation compared with not clipping on the incidence of collar rot. All clipped debris was removed from plants. (B) Effects of clipping and either leaving or removing clipped debris on incidence of collar rot. Clipped leaf tissue was macerated in a mortar and pestle and redistributed over the clipped plants. Bars with different letters are significantly different $(P=$ $0.05)$ according to the Waller-Duncan $k$-ratio test, $k=100$. age for disease development on tobacco seedlings. When plants less than 35 days old were inoculated, the incubation period increased beyond the typical 7- to 9-day period observed with older plants, such that plants were at least 42 days old before symptoms were observed. Plants 30 days old at inoculation had a 12-day incubation period, and plants 22 days old at inoculation had a 20-day incubation period. Plant characteristics responsible for this agerelated susceptibility have not been identified. Older seedlings develop a dense canopy, creating conditions that are known to be conducive for development of Sclerotinia diseases on other crops $(6,9,29)$. A closed canopy creates a favorable environment for ascospore survival, germination, and infection (7). We did not identify other host factors that may be responsible for the reduction in disease observed in the oldest seedlings. However, it is possible that the maturity of the stem tissues in older seedlings delayed colonization by $S$. sclerotiorum; infection in the oldest seedlings typically appeared as small cankers in the union of the petiole and the stem.

During the transplant production period, injuries other than clipping may occur. Based on the results from this study, injury may predispose tobacco seedlings to infection by $S$. sclerotiorum. The most commonly observed leaf injuries in commercial greenhouses are caused by poorly adjusted heating systems, propane gas leaks, insect pests, and cold temperatures. High incidence of collar rot was observed with heat $(65 \%)$ and chemical $(62 \%)$ injury, which resulted in necrotic areas on leaves. Wounded, dead, or senescent tissues are readily colonized by $S$. sclerotiorum ascospores $(3,10,18,22,25,34)$. We did not observe an effect of low temperature exposure on disease, but our low temperature exposure did not result in development of any symptoms on seedlings. Freezing injury on other plants may enhance disease caused by $S$. sclerotiorum $(18,36)$.

The inoculation method used in these experiments simulated a natural release and deposition of ascospores, and was highly effective in initiating disease on tobacco seedlings. This method also was effective in inoculation of cabbage plants (18), and it appears to be more effective and reliable than the use of ascospore suspensions $(19,23,27,33)$. In studies that used ascospore suspensions, an external carbon source was required to obtain high levels of disease, and while we observed an increase in disease in the presence of leaf extract, disease developed on older plants in the absence of leaf debris or leaf extract. If quantification of ascospores was necessary, ascospore deposition per unit area over time could be quantified using the semi-selective medium previously described for use in greenhouses $(13,15)$.

The results of this study indicated that seedlings between 38 and 52 days old are

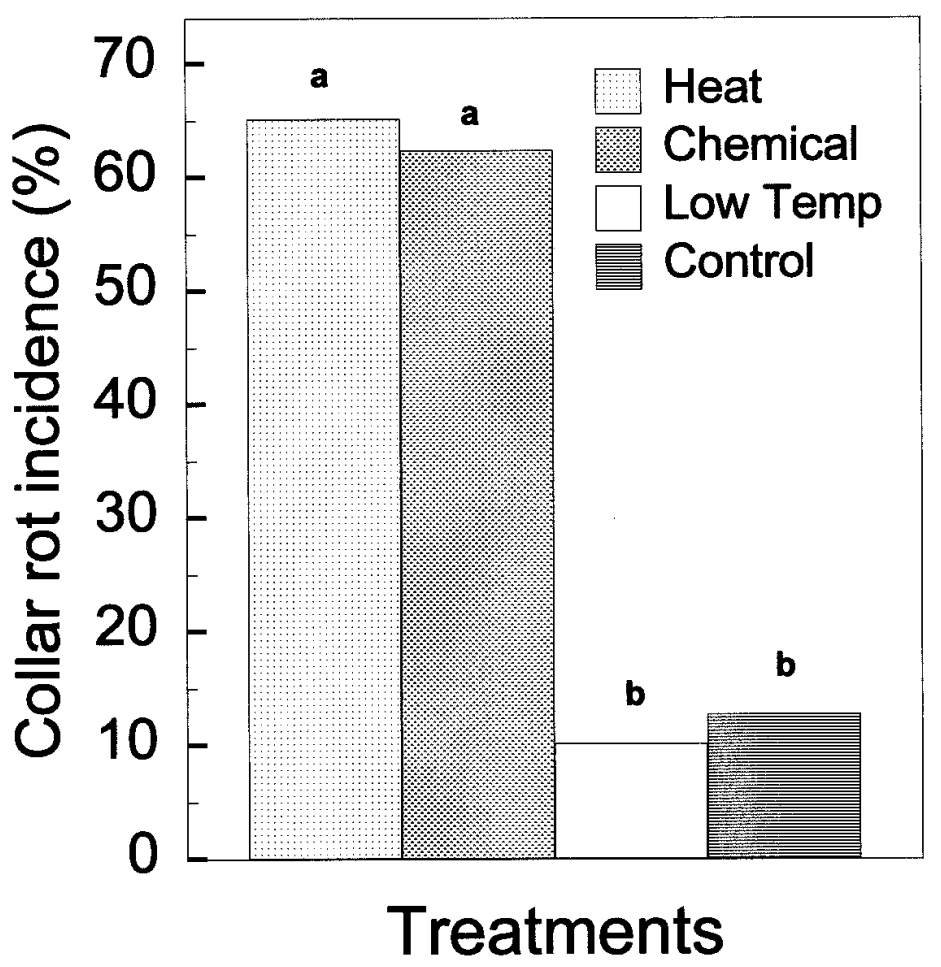

Fig. 4. Effect of plant injury on the incidence of collar rot of tobacco seedlings grown in float trays and inoculated with ascospores of Sclerotinia sclerotiorum. Heat injury was accomplished by blowing hot dry air on leaf surfaces with a hair dryer for $2 \mathrm{~min}$. Chemical injury was induced by spraying $5 \mathrm{ml}$ of methanol on seedlings. The low temperature treatment consisted of placing seedlings in a growth chamber at $4^{\circ} \mathrm{C}$ overnight. Bars with different letters are significantly different $(P=0.05)$ according to the Waller-Duncan $k$-ratio test, $k=100$. 
the most susceptible to collar rot. This time frame corresponds to the age at which clipping practices are initiated. Clipping practices alone had little effect on the incidence of collar rot, but the presence of leaf debris on the surface of tobacco seedlings increased the incidence of collar rot. Cultural practices that reduce production and deposition of leaf debris on plant surfaces and prevent or reduce necrotic injuries should be the foundation for reducing the incidence of collar rot of greenhouse-produced tobacco seedlings.

\section{LITERATURE CITED}

1. Abawi, G. S., and Grogan, R. G. 1975. Source of primary inoculum and effects of temperature and moisture on infection of beans by Whetzelinia sclerotiorum. Phytopathology 65:300-309.

2. Abawi, G. S., Polach, F. J., and Mollin, W. T. 1975. Infection of beans by ascospores of Whetzelinia sclerotiorum. Phytopathology 65:673-678.

3. Abawi, G. S., Provvidenti, R., Grogan, R. G., and Hunter, J. E. 1975. Predisposition of beans to infection by ascospores of Whetzelinia sclerotiorum prior to blossoming. Proc. Am. Phytopathol. Soc. 2:61.

4. Adams, P. B., and Tate, C. J. 1975. Factors affecting lettuce drop caused by Sclerotinia sclerotiorum. Plant Dis. Rep. 59:140-143.

5. Bedi, K. S. 1962. Effect of temperature in formation of sclerotia of Sclerotinia sclerotiorum (Lib.) de Bary. Indian Phytopathol. 15:55-60.

6. Blad, B. L., Steadman, J. R., and Weiss, A. 1978. Canopy structure and irrigation influence white mold disease and microclimate of dry edible beans. Phytopathology 68:14311437.

7. Caesar, A. J., and Pearson, R. C. 1983. Environmental factors affecting survival of ascospores of Sclerotinia sclerotiorum. Phytopathology 73:1024-1030.

8. Cook, G. E., Steadman, J. R., and Boosalis, M. G. 1975 Survival of Whetzelinia sclerotiorum and initial infection of dry edible beans in western Nebraska. Phytopathology 65: 250-255.

9. Coyne, D. P., Steadman, J. R., and Shwartz, H. F. 1977. Reaction of Phaseolus dry bean germplasm to Sclerotinia sclerotiorum. Plant Dis. Rep. 61:226-230.
10. Dillard, H. R., and Cobb, A. C. 1995. Relationship between leaf injury and colonization of cabbage by Sclerotinia sclerotiorum. Crop Prot. 14:677-682.

11. Gerlagh, M. 1987. The role of flowering in the infection by Sclerotinia sclerotiorum (white mould). Bull. SROP. 10:21-24.

12. Grogan, R. G., and Abawi, G. S. 1975. Influence of water potential on growth and survival of Whetzelinia sclerotiorum. Phytopathology 65:122-138.

13. Gutierrez, W. A., and Shew, H. D. 1995. A semi-selective medium for quantification of ascospores of Sclerotinia sclerotiorum. (Abstr.) Phytopathology 85:510.

14. Gutierrez, W. A., and Shew, H. D. 1995. Apothecia production of Sclerotinia sclerotiorum and Sclerotinia minor. (Abstr.) Phytopathology 85:1204.

15. Gutierrez, W. A., and Shew, H. D. 1998. Identification and quantification of ascospores as the primary inoculum for collar rot of greenhouse-produced tobacco seedlings. Plant Dis. 82:485-490.

16. Hartill, W. F. T., and Campbell, J. M. 1977. Effect of leaf maturation on the development of Sclerotinia/Botrytis complex of tobacco. N.Z. J. Agric. Res. 20:415-420.

17. Huang, H. C., and Kokko, E. G. 1992. Pod rot of dry peas due to infection by ascospores of Sclerotinia sclerotiorum. Plant Dis. 76:597-600.

18. Hudyncia, J., Shew, H. D., Cody, B. R., and Cubeta, M. A. 2000. Evaluation of wounds as a factor to infection of cabbage by ascospores of Sclerotinia sclerotiorum. Plant Dis. 84:316-320.

19. Jones, D. 1976. Infection of plant tissue by Sclerotinia sclerotiorum: A scanning electron microscope study. Micron 7:275-279.

20. Lucas, G. B. 1975. Diseases of Tobacco. 3rd ed. Biological Consulting Associates, Raleigh, NC.

21. Lumsden, R. D. 1979. Histology and physiology of pathogenesis in plant disease caused by Sclerotinia species. Phytopathology 69:890-896.

22. Morral, R. A., and Dueck, J. 1982. Epidemiology of Sclerotinia stem rot of rapeseed in Saskatchewan. Can. J. Plant Pathol. 4:161168.

23. Newton, H. C., and Sequeira, L. 1972. Ascospores as the primary infective propagule of Sclerotinia sclerotiorum in Wisconsin. Plant Dis. Rep. 56:798-802.

24. Phillips, A. J. L. 1994. Influence of fluctuating temperatures and interrupted periods of plant surface wetness on infection of bean leaves by ascospores of Sclerotinia sclerotiorum. Ann. Appl. Biol. 124:413-427.

25. Porter, D. M., and Powell, N. L. 1978. Sclerotinia blight development in peanut vines injured by tractor tires. Peanut Sci. 5:87-90.

26. Purdy, L. H. 1958. Some factors affecting penetration and infection by Sclerotinia sclerotiorum. Phytopathology 48:605-609.

27. Purdy, L. H. 1979. Sclerotinia sclerotiorum: History, diseases and symptomatology, host range, geographic distribution, and impact. Phytopathology 69:875-880.

28. Schwartz, H. F., Steadman, J. R., and Coyne, D. P. 1978. Influence of Phaseolus vulgaris blossoming characteristics and canopy structure upon reaction to Sclerotinia sclerotiorum. Phytopathology 68:465-470.

29. Sedum, F. S., and Brown, J. F. 1987. Infection of sunflower leaves by ascospores of Sclerotinia sclerotiorum. Ann. Appl. Biol. 110:275-285.

30. Smith, W. D., Fisher, L. R., and Boyette, M D. 2000. Transplant production. Pages 15-28 in: Flue-cured tobacco information. Bull. AG187. N.C. Coop. Ext. Ser. (revised).

31. Steadman, J. R., and Cook, G. E. 1974. A simple method for collecting ascospores of Whetzelinia sclerotiorum. Plant Dis. Rep. 58:190.

32. Sutton, D. C., and Deverall, B. J. 1983. Studies on infection on beans (Phaseolus vulgaris) and soybean (Glycine max) by ascospores of Sclerotinia sclerotiorum. Plant Pathol. 32:251-261.

33. Torres, J. A., and Moreno, R. 1991. Sclerotinia sclerotiorum: Epidemiological factors affecting infection on greenhouses in aubergine crops. J. Phytopathol. 132:65-74.

34. Tu, J. C. 1989. Modes of primary infection caused by Sclerotinia sclerotiorum in navy beans. Microbios 57:85-91.

35. Van der Berg, L., and Lentz, C. P. 1968. The effect of the relative humidity and temperature on survival and growth of Botrytis cinerea and Sclerotinia sclerotiorum. Can. J. Bot. 47:1007-1010.

36. Weingartner, P. D. 1990. White mold (Sclerotinia sclerotiorum) in Florida cabbage during 1989-1990. (Abstr.) Phytopathology 80:1026.

37. Willet, H. J., and Wong, J. A.-L. 1980. The biology of Sclerotinia sclerotiorum, Sclerotinia trifoliorum and Sclerotinia minor with emphasis in specific nomenclature. Bot. Rev. 46:101-165. 